\title{
Quantitative atomic resolution elemental mapping via absolute-scale energy dispersive X-ray spectroscopy
}

\author{
Z. Chen ${ }^{\mathrm{a}}$, M. Weyland ${ }^{\mathrm{b}, \mathrm{c}}$, X. Sang ${ }^{\mathrm{d}}$, W. Xu ${ }^{\mathrm{d}}$, J.H. Dycus ${ }^{\mathrm{d}}$, J.M. LeBeau ${ }^{\mathrm{d}}$, A.J. D’Alfonso ${ }^{\mathrm{e}}$, L.J. Allen ${ }^{\mathrm{e}}$, S.D. Findlay ${ }^{\mathrm{a}}$ \\ ${ }^{a}$ School of Physics and Astronomy, Monash University, Clayton, Victoria 3800, Australia \\ ${ }^{b}$ Monash Centre for Electron Microscopy, Monash University, Clayton, Victoria 3800, Australia \\ ${ }^{c}$ Department of Materials Science and Engineering, Monash University, Clayton, Victoria 3800, Australia \\ ${ }^{d}$ Department of Materials Science and Engineering, North Carolina State University, Raleigh, NC 27695, USA \\ ${ }^{e}$ School of Physics, University of Melbourne, Parkville, Victoria 3010, Australia
}

\begin{abstract}
Quantitative agreement on an absolute scale is demonstrated between experiment and simulation for two-dimensional, atomicresolution elemental mapping via energy dispersive X-ray spectroscopy. This requires all experimental parameters to be carefully characterised. The agreement is good, but some discrepancies remain. The most likely contributing factors are identified and discussed. Previous predictions that increasing the probe forming aperture helps to suppress the channelling enhancement in the average signal are confirmed experimentally. It is emphasised that simple column-by-column analysis requires a choice of sample thickness that compromises between being thick enough to yield a good signal-to-noise ratio while being thin enough that the overwhelming majority of the EDX signal derives from the column on which the probe is placed, despite strong electron scattering effects.
\end{abstract}

Keywords:

Scanning transmission electron microscopy (STEM), energy dispersive X-ray (EDX) spectroscopy, atomic-resolution mapping, elemental quantification.

\section{Introduction}

Atomic resolution elemental mapping via energy dispersive $\mathrm{X}$-ray (EDX) spectroscopy in scanning transmission electron microscopy (STEM) was achieved in 2010 [1-3]. Like the long-established high-angle annular dark field (HAADF) imaging mode, STEM EDX images are directly interpretable for qualitative analysis [4-6]. Also like HAADF [7], quantitative analysis of STEM EDX images requires accounting for the strong dynamical scattering, also called channelling, of the electron probe. The consequences of channelling for twodimensional (2D) maps can be broadly grouped into two categories: changes in the relative contrast and changes in the absolute-scale intensity. The former issue has been explored by Kothleitner et al. [8], who demonstrated good quantitative agreement between experiment and channelling-based simulations in the relative signal on different columns, even when, due to channelling, these ratios bear little resemblance to the true stoichiometry. Good agreement in relative contrast between simulation and experiments is also evident in the work of Forbes et al. [9] and Dycus et al. [10]. The issue of the absolute-scale of the signal was explored by Chen $e t$ al. [11], who demonstrated good agreement between experiment and simulation in the total number of X-ray counts recorded for an atomically-fine STEM probe scanned across a $\mathrm{SrTiO}_{3}$ specimen. The present paper unifies these two approaches, demonstrating absolute-scale comparison between experiment and simulation for atomic-resolution 2D EDX mapping.
Sufficient signal-to-noise to enable good quality atomic resolution 2D EDX maps is achieved here using a system with multiple, large-area, silicon-drift detectors (SDD) [4, 6, 12]. The increased complexity of this detector geometry necessitates accurate numerical modelling to determine effective detector solid angle and X-ray absorption in both the specimen and holder [13, 14]. Achieving good signal-to-noise for 2D images is more challenging than for scan-averaged spectra, especially from very thin specimens. While this can nominally be compensated for by using thicker samples, this may reduce the reliability of interpreting the signal on a column-by-column basis, since dynamical electron scattering can spread the probe appreciably beyond the column on which it is placed [15-17]. Here, experimental and simulation results are combined to produce some sense of the thickness range giving both reasonably good signal-to-noise and tolerably justifying simple columnby-column analysis.

\section{Methods}

\subsection{The experiment and materials}

EDX measurements were taken on an FEI Titan G2 with a probe aberration corrector (DCOR, CEOS $\mathrm{GmbH}$ ) operated at $200 \mathrm{keV}$. The EDX detection system comprises four windowless silicon-drift detectors (SuperX) giving a large collection solid angle, nominally $0.7 \mathrm{sr}[18,19]$ though, as discussed in Sec. 2.2 , the present analysis uses values calculated via the 
procedure given in Xu et al. [14] combining sample, holder and detector geometry and X-ray absorption. The high brightness X-FEG gun with monochromator affords much flexibility in the probe current - ranging from $\mathrm{pA}$ to $\mathrm{nA}$ - which was used to compromise between low beam damage and good counting statistics during EDX measurements. EDX maps were recorded using probe forming convergence semi-angles of 13.7, 19.5, 21.8 , and $45.7 \mathrm{mrad}$, with the probe current set to $105,92,89$ and $91 \mathrm{pA}$, respectively. Data were acquired by frame averaging, using the per frame automatic drift correction method in the Bruker Esprit acquisition software, confirming via inspection that specimen drift was less than half a unit cell between successive frames. The live time for each pixel in one scanning frame was $30 \mu \mathrm{s}$. More than 200 frames were acquired to get the final EDX maps, making the total acquisition time for each pixel about $8 \mathrm{~ms}$. The number of frames varied between datasets and was accounted for during the data analysis.

The specimen used was $\mathrm{SrTiO}_{3}$ oriented along the [001] zone-axis. The specimen was prepared with tripod wedge polishing followed by low-energy Ar ion-milling. EDX data were acquired from a range of different thicknesses. The thickness at each acquisition area was separately determined via positionaveraged convergent beam electron diffraction (PACBED) [20] using a probe forming convergence angle of $9.4 \mathrm{mrad}$ and an $\mathrm{L}^{2}$-normal comparison with simulation [11].

A normalized cross-correlation method [21] based on a $2 \times 2$ unit-cell area template in the simultaneously acquired HAADF image was used to post-process the raw EDX spectrum image data cube, averaging roughly fifty $2 \times 2$ unit-cell areas, to improve the signal-to-noise ratio prior to background subtraction. The background shape was determined via the fitting method of Huang [22] applied to the average EDX spectrum. The resultant background shape was then rescaled for subtraction from each spectrum in the spectrum image data cube. All quoted $\mathrm{X}$-ray counts represent the full shell signals for all identifiable peaks (e.g. $\mathrm{K}_{\alpha}+\mathrm{K}_{\beta}$ ) after subtraction of the Bremsstrahlung background.

\subsection{Theoretical framework}

The measured number of X-ray counts in each peak is taken as the absolute-scale for the experimental data. EDX simulations for the probe-position-dependent probability of core-shell ionization can be set on this same scale (Ref. [11] and the reference therein) via:

$$
N(\mathbf{R})=I T F_{\text {ion }}\left(\mathbf{R}, t, X_{\text {abs }}\right) \omega\left(\frac{\Omega}{4 \pi}\right) D_{\text {eff }} .
$$

Evaluating the number of X-ray counts for a particular peak, $N(\mathbf{R})$ as a function of probe position $\mathbf{R}$, requires determination of: $I$, the incident beam current; $T$, the probe live time; $F_{\text {ion }}$, the fraction of incident electrons causing ionization events (with thickness, $t$, dependent $X$-ray absorption correction, $X_{\text {abs }}$ ); $\omega$, the fluorescence yield; $\Omega$, the detector solid angle; and $D_{\text {eff }}$, the detector efficiency. The current was measured using the drift tube of a Gatan spectrometer. Fluorescence yields for the $\mathrm{Sr}-\mathrm{K}$, $\mathrm{Sr}-\mathrm{L}$ and Ti-K peaks were taken from Kahoul et al. [23]; that for the O-K peak was taken from Hubbell et al. [24]. The X-rayenergy-dependent detector efficiency was taken from Schlossmacher et al. [25]. To compare experiments carried out with different currents and total acquisition times, the number of $\mathrm{X}$ ray counts was normalized by the live time and the beam current to give unit of counts/s/nA.

The fraction of incident electrons causing ionization events that can subsequently produce the $\mathrm{X}$-ray peak under consideration, $F_{\text {ion }}\left(\mathbf{R}, t, X_{\text {abs }}\right)$, must account for the dynamical elastic and thermal scattering of the electron probe. This was calculated based on the quantum excitation of phonons (QEP) model [26] as implemented in the $\mu$ STEM simulation suite [27] using the equation

$$
F_{\text {ion }}\left(\mathbf{R}, t, X_{\text {abs }}\right)=\int_{0}^{t} \int I\left(\mathbf{R}, \mathbf{r}_{\perp}, z\right) V_{\text {eff }}\left(\mathbf{r}_{\perp}\right) X_{\text {abs }}(z) d \mathbf{r}_{\perp} d z,
$$

where $t$ denotes specimen thickness, $I\left(\mathbf{R}, \mathbf{r}_{\perp}, z\right)$ denotes the probe electron intensity inside the sample, $V_{\text {eff }}\left(\mathbf{r}_{\perp}\right)$ denotes the effective scattering potential for ionization, and $X_{\text {abs }}$ accounts for X-ray absorption. For the four detector system, the contributions to X-ray absorption from both the specimen and holder shadowing depend on the position of the specimen and on the orientation of the specimen holder, which it was necessary to vary slightly at each region of varying thickness for best onaxis specimen alignment. The fraction of X-rays reaching the detector can be expressed as an integration over the emission angles directed towards the detector of a depth-dependent function, $X_{\text {specimen }}(\theta, \phi, z)$, describing the fraction of X-rays generated at depth $z$ transmitted through the specimen, and a function, $X_{\text {holder }}(\theta, \phi)$, describing the fraction of X-rays transmitted through or around the specimen holder:

$$
X_{\mathrm{abs}}(z)=\frac{1}{\Omega} \iint_{\Omega} X_{\text {specimen }}(\theta, \phi, z) X_{\text {holder }}(\theta, \phi) \sin \theta d \theta d \phi .
$$

The procedure for describing the sample/holder/detector geometry and calculating the $\mathrm{X}$-ray absorption is given in $\mathrm{Xu}$ et al. [14]. (See Slater et al. [28] for an alternative approach.) For continuity with Chen et al. [11], Eqs. (1) and (3) separate the physical solid angle spanned by the detectors from the effects of absorption in specimen and holder, whereas Xu et al. combine these into an effective solid angle [14]. If X-ray generation is uniform through depth, the $\mathbf{r}_{\perp}$ and $z$ integrations in Eq. (2) decouple and the absorption correction reduces to multiplication with an average absorption factor. In the presence of channelling, $\mathrm{X}$-ray generation is not usually uniform with depth $[9,11]$. However, when the large incoherent effective source size of the present experiment (discussed below) is taken into account, the generation of X-rays is sufficiently uniform with depth to allow this simplifying approximation - the error is on the order of $1 \%$ for peak values and a fraction of that for mean values - which is therefore used in all calculations in this manuscript.

Since the coherent aberrations are well-balanced, the probe can be modelled as being aberration-free. The possible exception is defocus. In the experiment, focus was manually adjusted to maximise the ADF image contrast. Simulations therefore 
assume the beam waist to be at the crystal entrance surface as this tends to maximize the ADF image contrast for all but the thinnest of crystals [29]. The broadening caused by the combined effects of finite gun size, instabilities in the probeforming optics, and planar sample drift during the acquisition are encapsulated into an incoherent effective source distribution. This distribution is assumed to be Gaussian, though recent work suggests a more detailed characterisation of the distribution can be important [30-32]. It was found that a full-widthhalf-maximum of $2.1 \AA$ for the Gaussian gave the best fit to the EDX map contrast - defined as the integrated number of counts from an atomic column divided by the mean counts in the full image, which ratio is independent of the absolute scale - over the full range of thicknesses. This relatively large value is a consequence not only on the gun conditions chosen for EDX acquisition but also of the acquisition procedure whereby multiple EDX scanning frames were collected and averaged after cross-correlation alignment using simultaneously acquired HAADF images. This latter effect is non-trivial: for a single frame HAADF image recorded during the EDX acquisition we estimate a Gaussian effective source full-width-half-maximum value of $1.6 \AA$. Both values seem largely independent of probeforming aperture angle, suggesting this source size is limited more by instabilities than source brightness. There was some evidence of vertical specimen drift during the long EDX acquisition, amounting to an effective variation in focus. To the extent that this was consistent between acquisitions, its effect is approximately included in the effective source size.

\section{Experimental results}

\subsection{Atomic resolution mapping on an absolute scale}

Fig. 1 compares experimental and simulated EDX signals for the Sr-K, Ti-K and Sr-L peaks using a $19.5 \mathrm{mrad}$ probe-forming convergence angle. The left side compares $2 \mathrm{D}$ maps for three selected thicknesses. The images in each pair of experimental and simulated 2D EDX maps use colour maps on the same absolute scale. Subject to noise, both the absolute signal and the contrast are seen to be in largely good agreement. Despite the low signal-to-noise ratio for the very thin area, $55 \AA$, atomic resolution contrast is clearly seen in those experimental $\mathrm{Sr}-\mathrm{K}$, Ti-K and Sr-L peak EDX maps.

For a more quantitative comparison, the central column of Fig. 1 shows corresponding line profiles, vertical averages from the white rectangular regions shown on the maps on the left in Fig. 1. For the most part this reinforces the agreement seen in the 2D maps: for the thicker samples, $176 \AA$ and $387 \AA$, the intensity distributions in the experimental and simulation maps are quite similar, and the width, maximum and minimum in the line profiles show acceptable agreement. However, it also reveals some discrepancies. The line profiles for the thinnest sample, where the signal-to-noise ratio is very low, show a relatively large discrepancy, with lower count rates in the experiment than in the simulation. (The discrepancy is smaller for the Sr-L peak than the other two, but this may be an example of compensating errors since, as shall be shown, the $\mathrm{Sr}-\mathrm{L}$ signal seems to be systematically underestimated in the simulation.) The line profiles for the Sr-L map at $176 \AA$ and for the Ti-K and Sr-L maps at $387 \AA$ also show notable discrepancies.

As has been well understood in quantitative HAADF imaging [33], quantitative comparison of 2D EDX maps depends on an adequate modelling of the coherent and incoherent aberrations in the probe. The extent to which uncertainties in modelling the aberrations might be responsible for the discrepancies between experiment and simulation can be gauged from quantities which are insensitive to these values. To this end, the plots on the right hand side in Fig. 1 show the mean, column and minimum intensities for a wide range of thicknesses. The mean signal should be strictly independent of aberrations due to the process of averaging over the unit cell [20,34]. The column and minimum signals - which, to reduce shot noise effects, constitute the average signal within a radius of $1.0 \AA$ around the atomic column and minimum position, respectively, as determined from the simultaneously acquired HAADF image - should also be relatively insensitive to residual aberrations [35]. On the whole, the agreement is reasonable, comparable to previous work [11]. It must be noted, however, that the discrepancies are larger than can be explained by fluctuations due to counting statistics alone. Note that the discrepancy between experiment and simulation for the column and minimum counts has a similar trend to that of the mean counts. This suggests that the Gaussian shape and source size used, which, recall, was determined by a ratio metric which was insensitive to the absolute value of the mean, gives a reasonable estimate of the source size broadening. Moreover, the discrepancies previously noted in the line profiles for the Sr-L map at $176 \AA$ and the Ti-K and Sr-L maps at $387 \AA$ are commensurate with the discrepancies evident in the mean intensities. This suggests these differences should not be attributed to any particular shortcoming of modelling the probe intensity, as the mean signal is insensitive to this.

The mean signals, being robust to noise and aberrations, are a fruitful metric for seeking a better understanding of possible causes for the remaining discrepancies. That the experimental results for the Sr-L and Ti-K peaks for the thicknesses around 300 and $400 \AA$ are larger than the simulated results may indicate that the sample thickness is being underestimated. This is possible because the minima in the $\mathrm{L}^{2}$-norm used for thickness determination from the PACBED patterns are broader for thicker samples, making the thickness estimation less precise, and also because the PACBED analysis is sensitive only to the crystalline thickness, which is usually slightly smaller than the sample thickness. That the experimental mean EDX counts for the $\mathrm{Sr}-\mathrm{K}$ peak are conversely overall about $10 \%$ smaller than those in the simulation, suggests a systematic error. In particular, since the experimental Sr-K signal is lower than simulation whereas if anything the Sr-L signal is slightly higher than simulation, the explanation should not lie in amorphous surface layers or sample contamination, but rather in one of the X-rayenergy-dependent factors in Eqs. (1)-(3). The most likely explanation is the detector efficiency taken from the literature: the efficiency curve is steep in the vicinity of the Sr-K X-ray energies, so relatively small variations in the overall form of the 
(a)

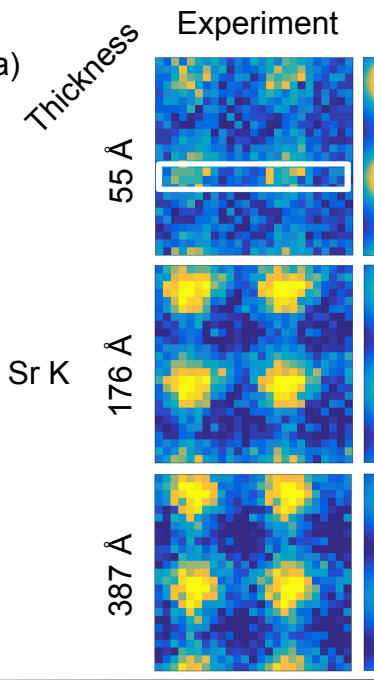

(b) TiK
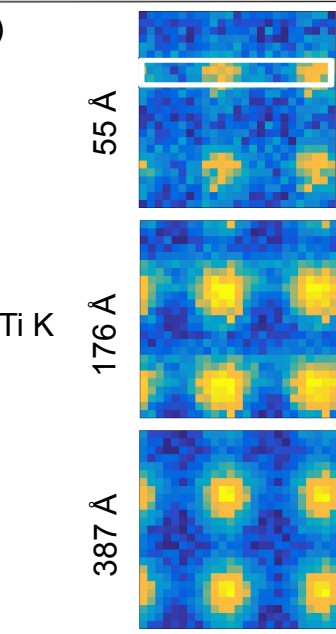

(c)
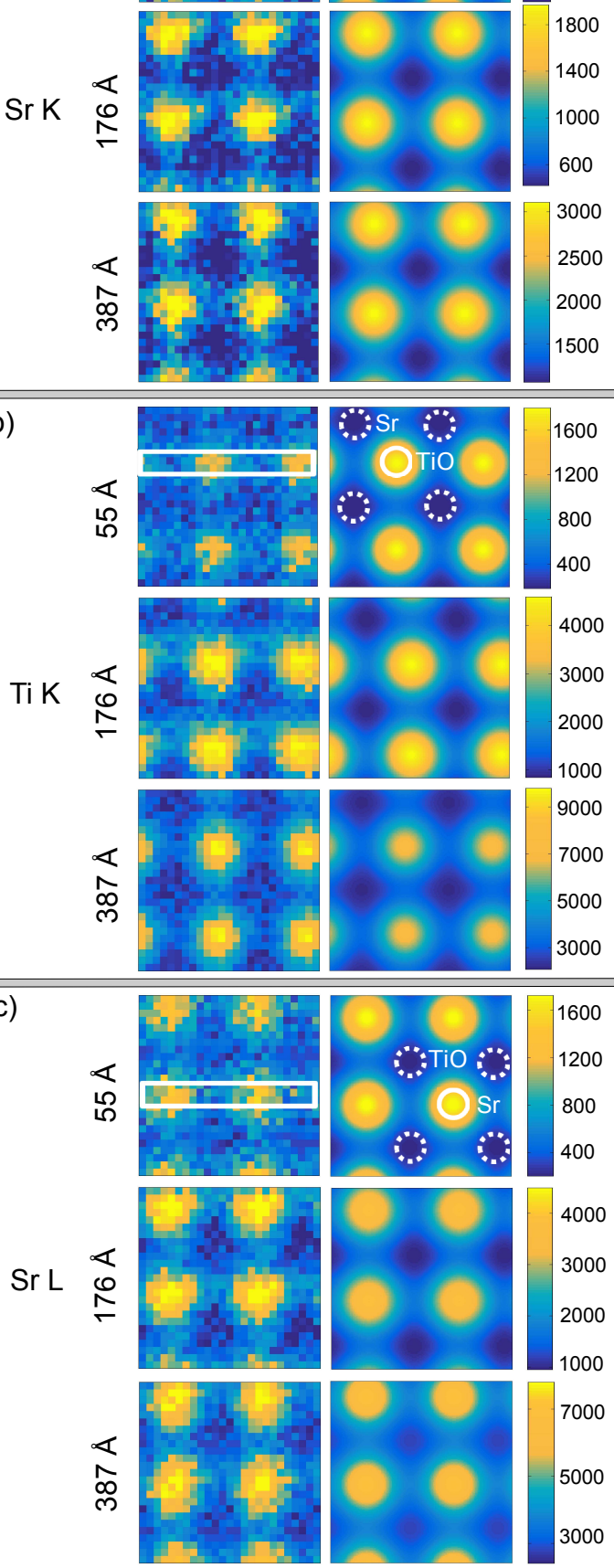

Line profile
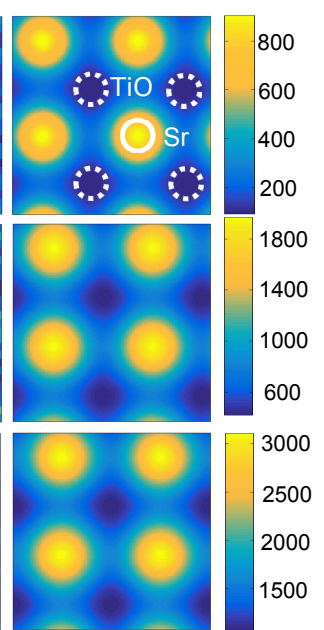

- Experiment - Simulation
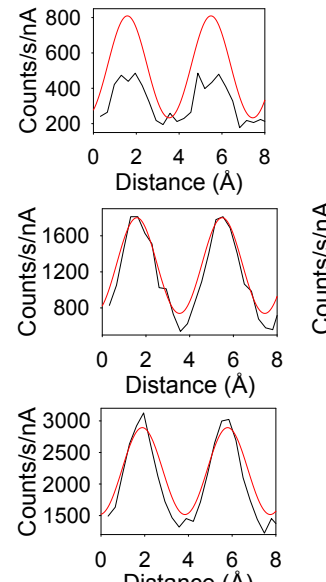
Distance $(\AA)$
Column, mean, minimum

Sr K 19.5 mrad

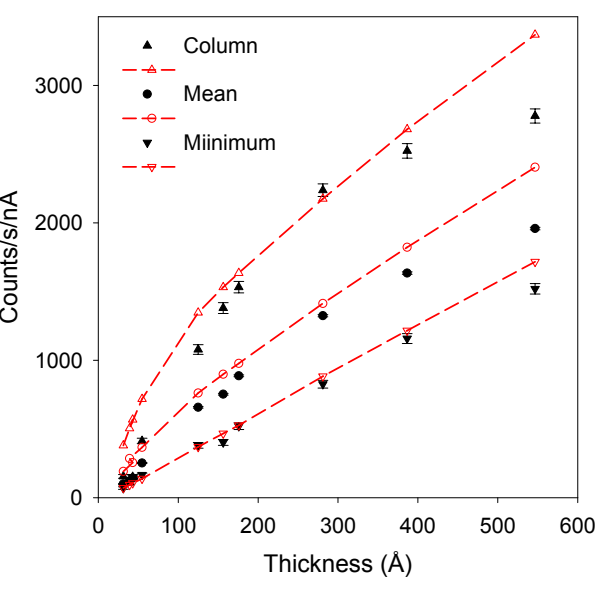

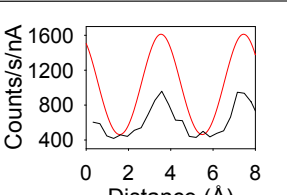
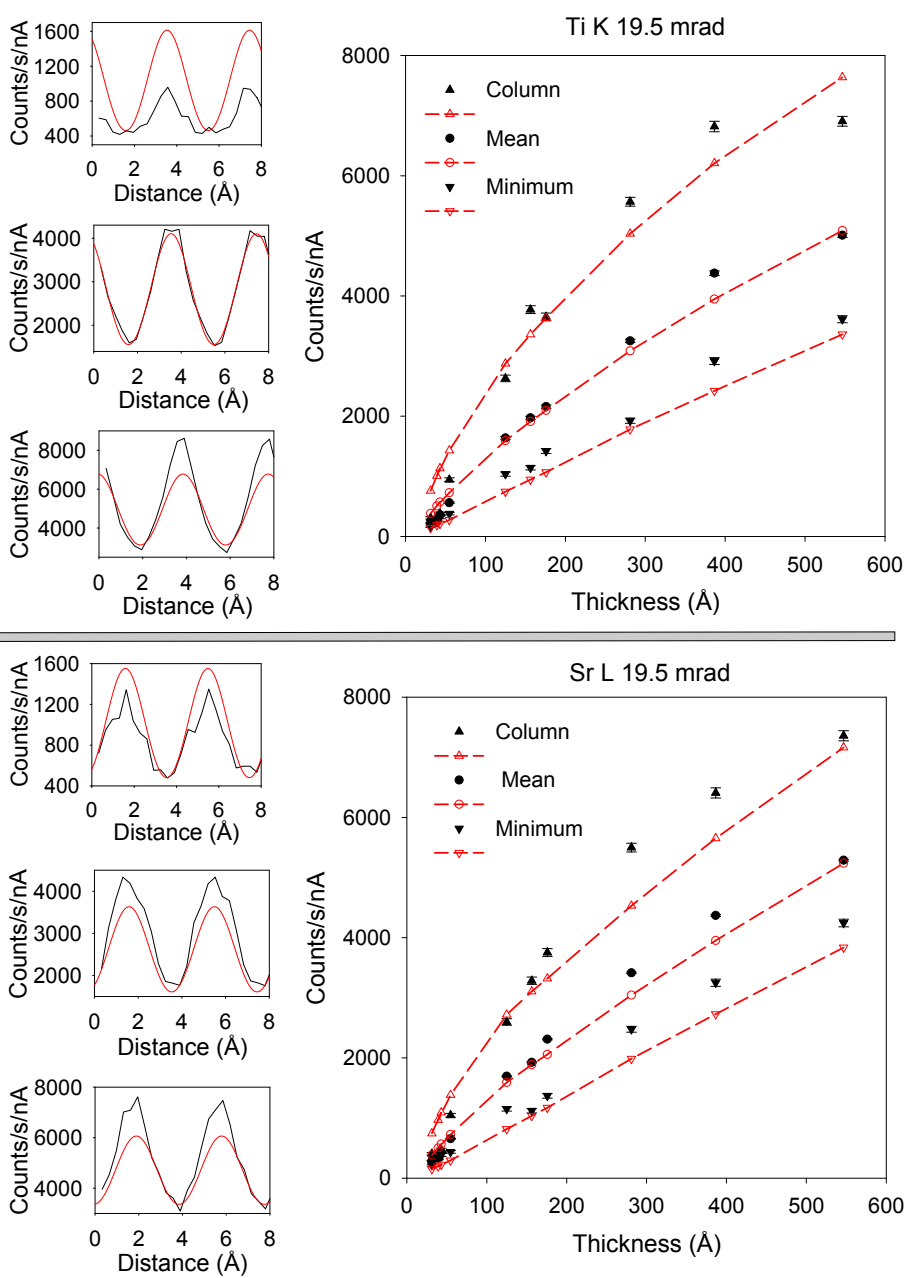

Figure 1: Absolute-scale comparison of experimental and simulated EDX signals for the (a) Sr-K, (b) Ti-K, and (c) Sr-L peaks. Left: 2D maps are shown for three different thicknesses: $55 \AA$, $176 \AA$, and $387 \AA$. The convergence angle is 19.5 mrad. The incoherent effective source distribution was taken to be a Gaussian with $2.1 \AA$ full-width-half-maximum. Centre: line profiles of the intensity averaged across the $1.6 \AA$ wide white rectangular regions shown on the $2 \mathrm{D}$ maps. Right: comparison between experimental (black solid symbols) and simulated (red open symbols and dashed lines) column, mean and minimum number of X-ray counts in the 2D EDX maps as a function of specimen thickness. The column and minimum counts constitute the average signal within a radius of $1.0 \AA$ around the atomic column and minimum positions, respectively. The dashed lines are only guides to the eye: slight variations in holder orientation used for data from the different

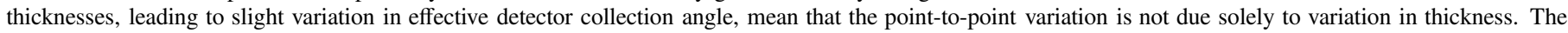
error bars given on the experimental data are the contribution from counting statistics alone. 
efficiency curve could lead to appreciable systematic errors for these peaks. Other contributing factors may include the correction due to the effective solid angle and the X-ray absorption (due to sample holder occlusion). The contribution from the secondary fluorescence yield, which is usually very small for these quite thin specimens with very small angle wedge shape [36], may also have an effect.

The right hand plots in Fig. 1 obscure discrepancies for thin specimens, but the discrepancies seen in the line profiles again prove to be associated with discrepancies more in the robust metric of the mean signal than in the relative contrast. It may again be that thickness determination introduces some uncertainty, since there is little structure present in the PACBED patterns for very thin samples and modest errors of a nanometer or so constitute a large fractional change in material present. In addition, the adverse effects of beam damage - the total dose during acquisition was about $5 \times 10^{7} \mathrm{e}^{-}$per $\AA^{2}-$ are proportionally larger for very thin specimens. In the data presented, reductions in beam current and post-selection of suitable data sets as judged by comparing HAADF images acquired both before and after the EDX maps were used to mitigate against this effect (example before and after images are shown in Figs. S1-S3 of the supplementary information). Beam damage could have been further reduced for still lower beam currents [10], but at the expense of further reducing the count rate.

\section{2. $O-K$ EDX maps}

The background fitting method of Huang [22] was not deemed reliable in the vicinity of the $\mathrm{O}-\mathrm{K}$ peak due to the proximity of nearby peaks, e.g. the C-K peak. Rather, a linear background was fitted from the minimum positions in the spectrum before and after the overlapping $\mathrm{O}-\mathrm{K}$ and Ti-L peaks. The $\mathrm{O}-\mathrm{K}$ and Ti-L peaks were then separated via a linear least-squares fitting procedure in which the amplitude and width of three Gaussian functions were allowed to vary while their energy positions were fixed to the known locations of the Ti- $\mathrm{L}_{1}, \mathrm{Ti}-\mathrm{L}_{\alpha \beta}$ and $\mathrm{O}-$ $\mathrm{K}$ peaks. This method for separation of Ti-L and O-K peaks requires much better signal-to-noise of the spectrum. Therefore, to increase the fitting confidence, a $5 \times 5$ pixel box average was applied to the experimental EDX data cube prior to the background subtraction and Gaussian fitting procedure in producing the maps, column, mean and minimum EDX counts of $\mathrm{O}-\mathrm{K}$ peak shown in Fig. 2. The difference in channelling along the mixed $\mathrm{TiO}$ and pure $\mathrm{O}$ columns [9] and the large effective source size make the two different $\mathrm{O}$-bearing columns difficult to clearly identify in the 2D maps of Fig. 2(a). Nevertheless, column signals from two different column positions are given in Fig. 2(b) on the basis of the known $\mathrm{SrTiO}_{3}$ structure.

The experimental and simulated 2D maps in Fig. 2(a) have colour scales set to the individual image maxima and minima, revealing them to have similar contrast. However, their absolute scale intensity differs appreciably. Fig. 2(b) reveals that the mean experimental intensity is about $10-20 \%$ larger than that from simulation. The comparison of simulated and experimental column and minimum intensity shows a similar discrepancy to the mean intensity. As per the discussion of the Sr-K peak, this again suggests a systematic error. Possible origins of the discrepancy include the detector efficiency (the efficiency curve is again quite steep in the vicinity of the O-K X-ray energies), the effective solid angle accounting for X-ray absorption (which is about $50 \%$ smaller for $550 \AA$ thickness than it is for the thinnest region), secondary fluorescence yield, and the reliability of the peak separation and background subtraction. In addition, unavoidable hydrocarbon contamination, which is usually rich in oxygen, is also a possible factor (see supplementary information for EDX spectra showing the presence of the $\mathrm{C}-\mathrm{K}$ peak as indicative of hydrocarbon contamination).

\subsection{Effects of the probe-forming convergence angle}

The results presented thus far use a $19.5 \mathrm{mrad}$ probe convergence angle, a typical value for atomic resolution imaging in aberration-corrected STEM, but it is worth considering here the relative merits of different choices of convergence angle for quantitative 2D EDX mapping. One possible criterion is direct, quantitative interpretability, to which dynamical electron scattering is generally a hindrance [8,9]. Chen et al. [11] previously predicted that the mean EDX signal tends towards the "non-channelling" result, i.e. the idealization in which dynamical electron scattering does not occur, in the limit of large probe-forming aperture. The comparison shown in Fig. 3 between experiment and simulation for the mean EDX intensity for different probe forming apertures together with the predicted intensity from the non-channelling condition confirms this: the result using a very large convergence angle, $45.7 \mathrm{mrad}$, is the closest to the ideal non-channelling case. (As per previous sections, some systematic discrepancies are evident in Fig. 3 , especially in the $\mathrm{Sr}-\mathrm{K}$ and $\mathrm{O}-\mathrm{K}$ peaks, reinforcing the notion that they arise from some inaccuracy of one of the multiplicative factors in Eq. (1).)

The advantage of the non-channelling limit is that it is the domain in which the traditional Cliff-Lorimer "k-factor" analysis [37] is valid, which enables conversion between measured intensity ratios and elemental concentration ratio on the basis of the ratio of signals expected for isolated atoms. By contrast, as shown by Chen et al. [11], in the presence of channelling the k-factor analysis needs to be augmented by a thickness-dependent channelling correction. Fig. 3 shows the non-channelling limit is not reached: there are $20 \%$ more EDX counts in the $45.7 \mathrm{mrad}$ case than in the ideal non-channelling result. However, linearity of the number of X-ray counts with thickness is better than at lower aperture sizes, meaning that the thickness-dependence of the needed channelling correction is weak. It should therefore be possible to reliably use measurements or simulations from certain known thicknesses to predict the counts for other thicknesses, facilitating EDX quantification in real material systems.

While very large probe convergence angles may simplify assessment of average composition, they are not necessarily favourable for atomically resolved 2D EDX mapping. Intriguingly, Fig. 4 shows that atomically-resolved contrast can be seen in the HAADF and EDX maps for the 45.7 mrad convergence angle case, despite the aberrations present in this probethe upper limit angle of flat phase area in the Ronchigram, i.e. 
(a)

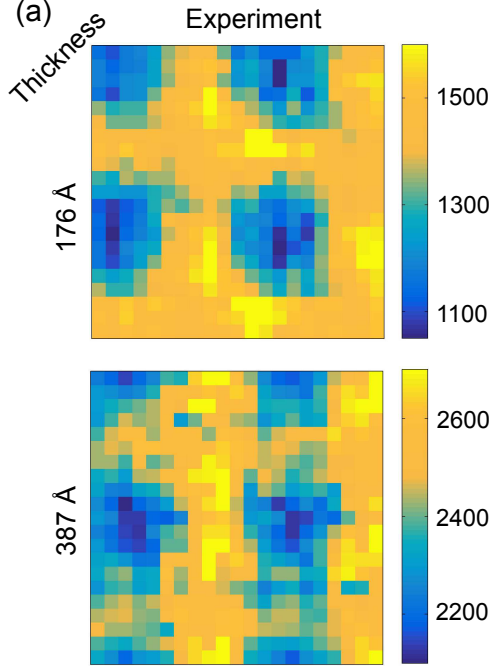

Simulation
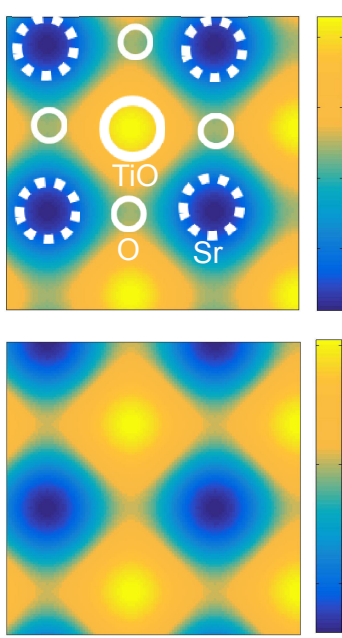

(b)

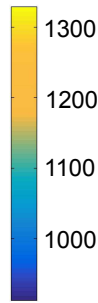

2100

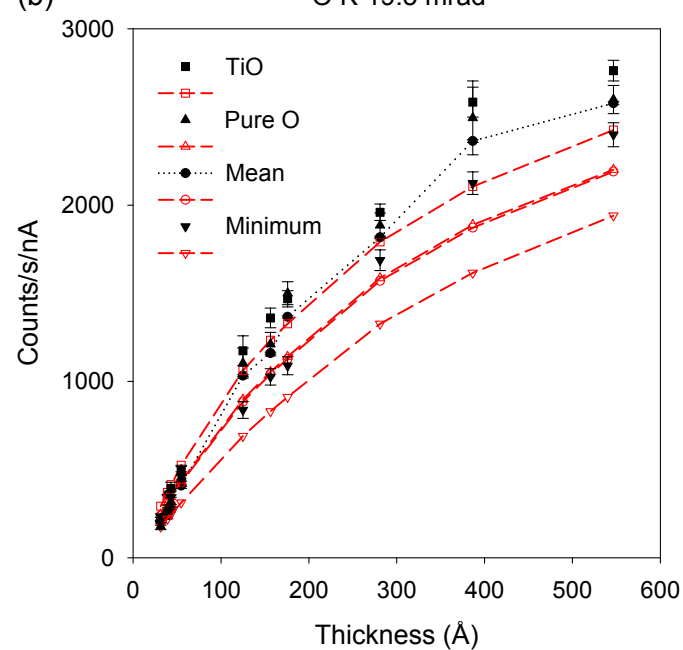

Figure 2: Comparison between experimental and simulated O-K EDX signal in (a) 2D mapping and (b) mean, column and minimum numbers of X-ray counts. Note that there are two different O-bearing columns: mixed TiO columns (square symbols in (b)) and pure O columns (triangular symbols in (b)). Black solid symbols and red open symbols represent the experimental and simulated data, respectively.

(a)

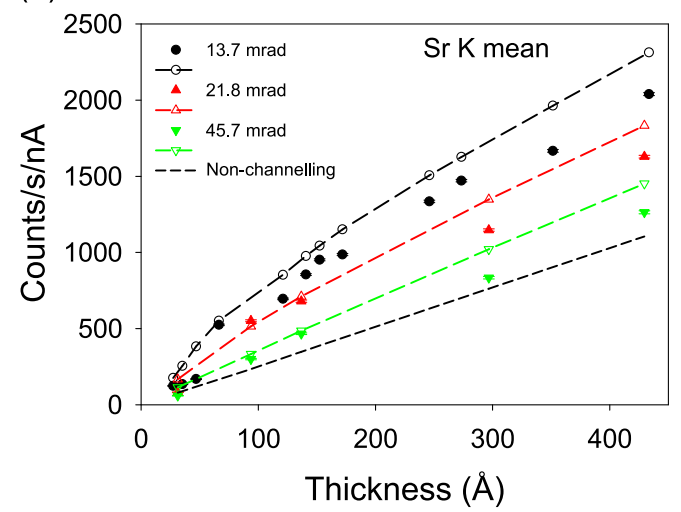

(c)

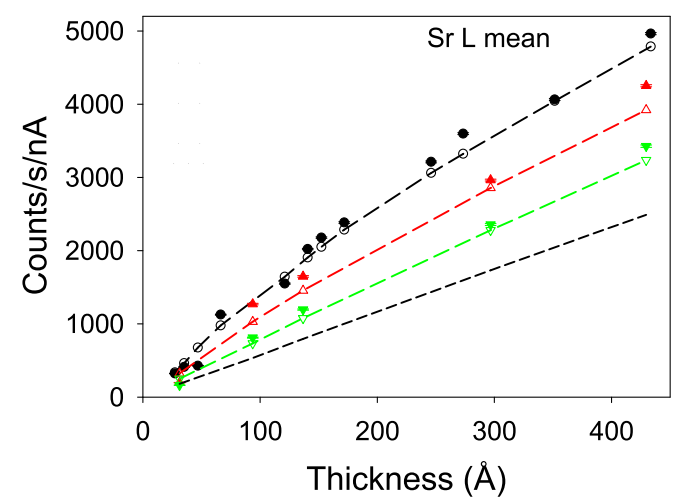

(b)

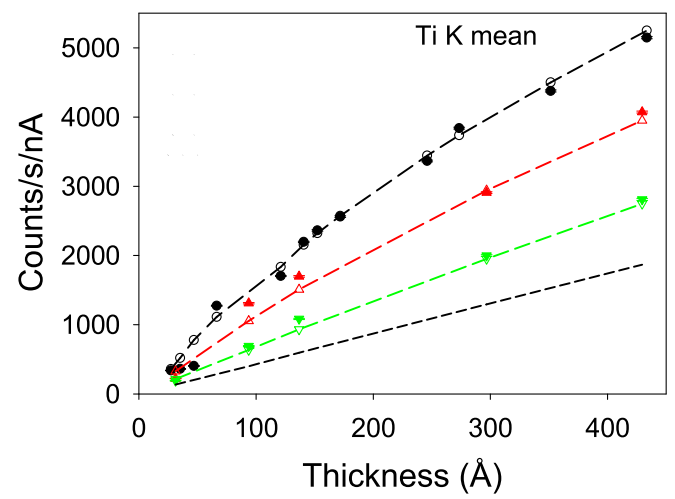

(d)

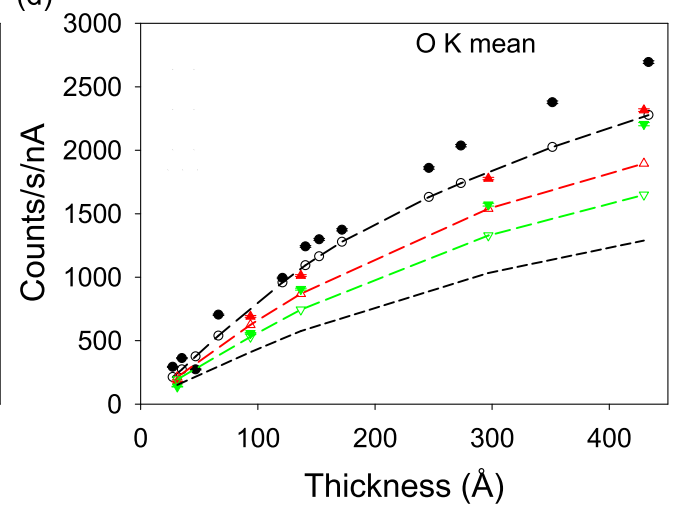

Figure 3: Comparison between experimental (filled symbols) and simulated (open symbols and dashed lines) mean intensities as a function of sample thickness for the (a) Sr-K, (b) Ti-K, (c) Sr-L, and (d) O-K peaks. Results are shown for three different convergence angles - $13.7 \mathrm{mrad}$ (black), $21.8 \mathrm{mrad}$ (red) and $45.7 \mathrm{mrad}$ (green) - along with the simulated non-channelling prediction (black short-dashed line).

that with a phase shift less than $\frac{\pi}{4} \mathrm{rad}$, is only $24 \mathrm{mrad}$. Nevertheless, while the presence of atomically-resolved features of- fers some sense of where the probe is on the specimen, this is not to say that column-by-column analysis is possible here. 
Rather, probe spreading [38] and the probe tails arising from aberrations means the signal at any nominal probe position actually contains a significant admixture of signal from a wider region of the sample. Since the motivation for high-resolution 2D mapping is usually to reliably map localized features, smaller probe-convergence angles are to be preferred, even though they will necessitate channelling effects be taken into account for quantitative work.

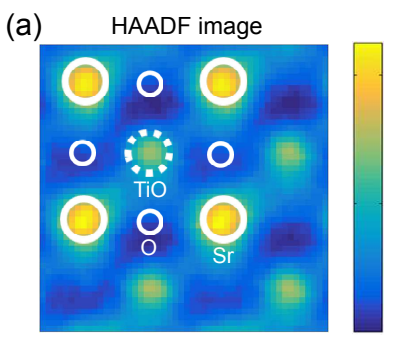

(c)

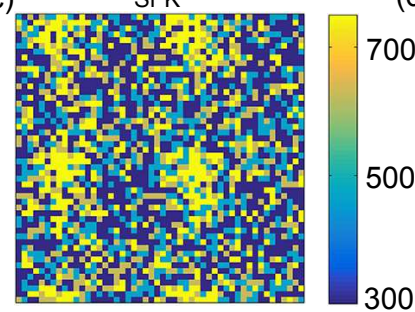

(b)

(d)

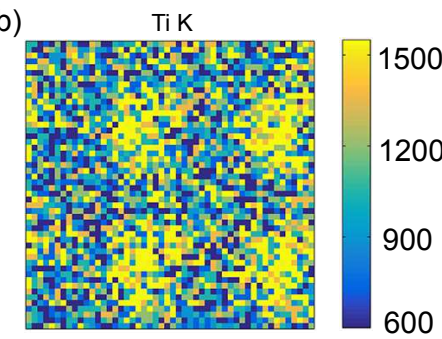

Sr L

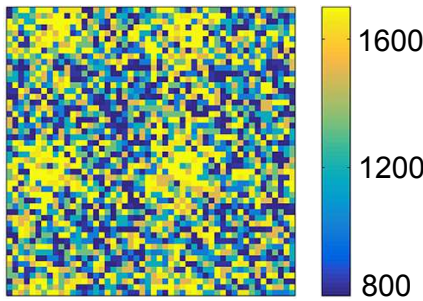

Figure 4: (a) HAADF image, (b) Ti-K map, (c) Sr-K map, and (d) Sr-L map for a $45.7 \mathrm{mrad}$ probe-forming convergence angle and a $137 \AA$ thick specimen.

Fig. 3 shows that channelling, while hindering quantitative analysis, in some respects aids qualitative analysis: for the same beam current and acquisition time, the mean number of EDX counts increases for decreasing aperture size. Large signal means better counting statistics and so improved signal-tonoise. The question of optimizing probe convergence angle to achieve both good contrast in the atomic-resolution image and good overall signal-to-noise is addressed in Dycus et al. [10] and will not be repeated here. However, recognising that the lower probe convergence angles which strike this balance are those for which dynamical electron scattering effects are pronounced, the question of reliably mapping local features, i.e. column-by-column interpretability in atomic-resolution imaging, should be examined further.

\section{Reliability of column-by-column analysis for atomic res- olution STEM EDX}

As seen in Fig. 1, quantification of EDX maps for thin samples presents challenges: the EDX signal from a thin specimen is quite weak, meaning the statistical uncertainty is large, it is difficult to reliably determine the thickness, and surface oxides, amorphous layers and beam damage constitute a larger fraction of the recorded signal. These limitations can all be overcome by using thicker specimens. However, as has been much emphasised in the literature [15-17], dynamical probe scattering and spreading means that the reliability of column-by-column analysis reduces with increasing specimen thickness because an increasing fraction of contributions to the recorded signal may originate through scattering events from columns other than that on which the probe was placed. This is particularly acute for HAADF [15-17], because all atomic species contribute to the signal, and electron energy loss spectroscopy [39], because of the delocalization of the signal. The issue has been less explored for atomic resolution EDX mapping. Nevertheless, it is anticipated that atomic scale quantification will be most reliable for intermediate specimen thicknesses that compromise between being thick enough to obtain enough X-ray counts to give an adequate signal-to-noise ratio and being thin enough that column-by-column analysis is not undermined by probe spreading.

To visualize probe spreading in the present case study, Fig. 5 shows the electron intensity distribution in both through-depth and in-plane cross-sections within the $\mathrm{SrTiO}_{3}$ crystal. While the intensity has been clipped to enhance the visibility of the structure for low intensities, significant beam spreading can clearly be seen for both the $\mathrm{Sr}$ and $\mathrm{TiO}$ columns. Following Eq. (1), contributions to EDX come from the overlap integral between the probe intensity and the effective scattering potential for ionization. The latter is highly localized for EDX, whereas the former can be quite diffuse away from the column under the probe. The visual impression of probe spreading given by Fig. 5 therefore does not tell the full story: it accurately reflects the sizeable fraction of electron density diffusely spreading to significant distances from the column on which the probe is placed, but does not readily allow one to judge what fraction of this intensity is in close proximity to atomic columns, which is what actually determines how much those columns contribute to the total EDX signal.

Inner shell ionization can, to an excellent approximation, be treated on an atom-by-atom basis [40]. In simulation, it is therefore possible to unambiguously decompose the effective scattering potential $V_{\mathrm{eff}}\left(\mathbf{r}_{\perp}\right)$ in Eq. (2) into a sum over each individual atom. Consequently, the total signal can likewise be unambiguously decomposed into the contribution from each individual atom, or, more usefully for the present purpose, each individual column. In this way, the EDX signal for a given probe position can be decomposed to identify how much of that signal comes from the column underneath the probe, and how much from more distant columns as a result of probe scattering and spreading. The results of this exploration are shown in Fig. 6.

The case of Sr K-shell ionization is given in Fig. 6(a) for the probe placed directly above a $\mathrm{Sr}$ column. This shows that for specimens thinner than around $250 \AA$, more than $95 \%$ of the signal derives from the column directly under the probe, whereas by a thickness of $600 \AA$ more than $15 \%$ derives from columns other than that directly under the probe. (For Ti-K case, not shown here, more than $95 \%$ of the signal derives from the column directly under the probe up to a thickness of $400 \AA$.) Beyond about $250 \AA$, the absolute contribution from the probe under the column saturates - almost all the gain in signal obtained by using still thicker samples comes from columns other than that on which the probe is placed. Indeed, the additional contributions come from the combined effect of columns a sig- 


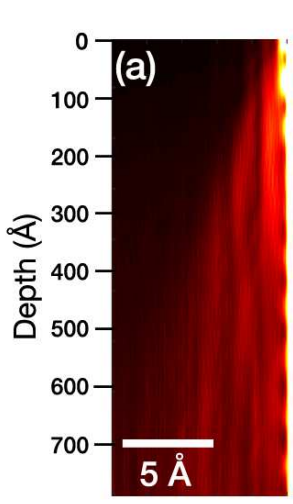

(e)

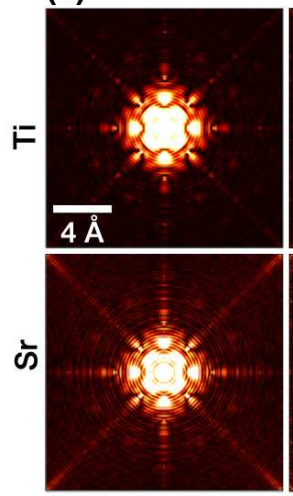

$150 \AA$
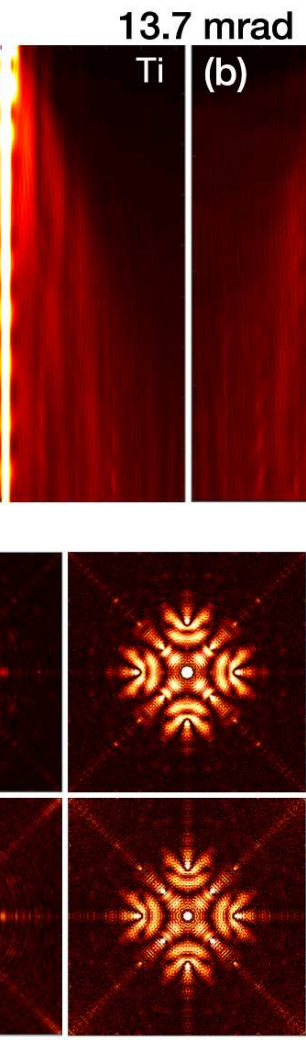

$300 \AA$

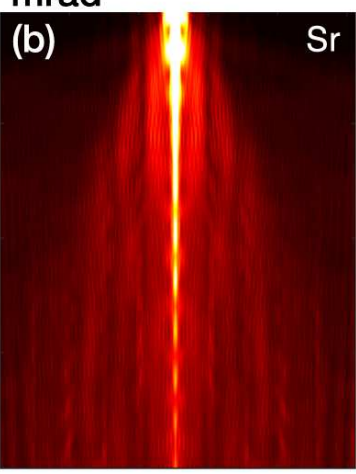

$\mathrm{Sr}$
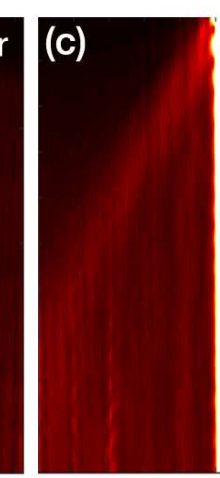

(f)

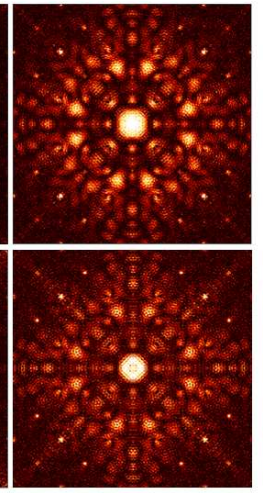

$600 \AA$

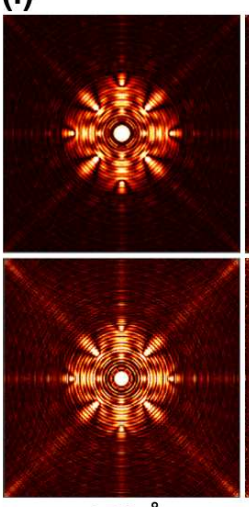

$150 \AA$
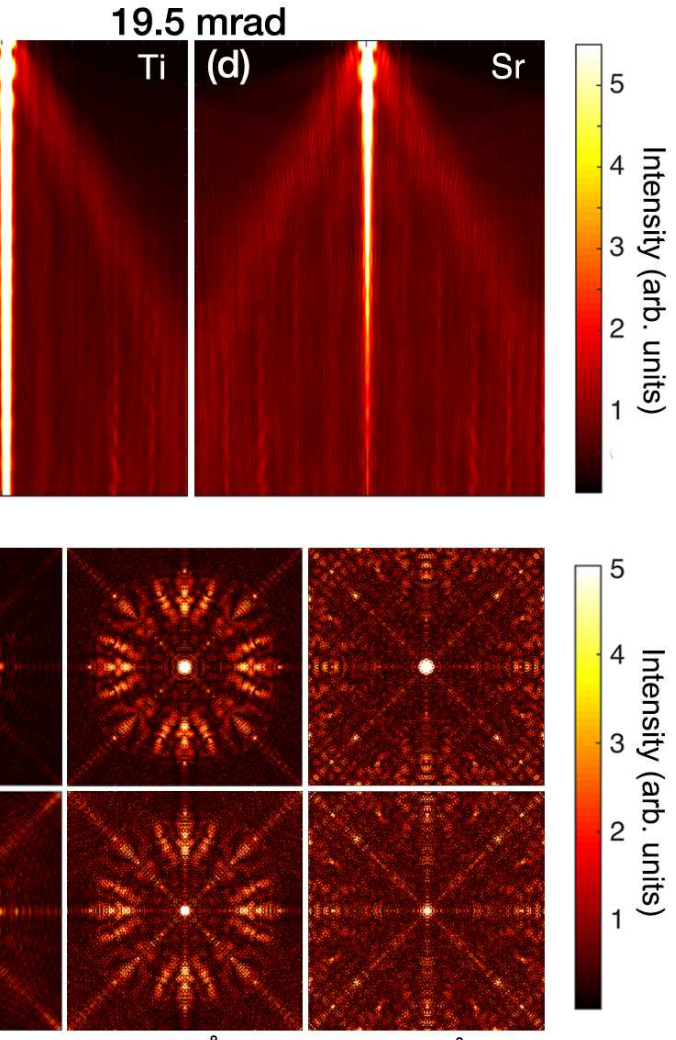

$300 \AA$

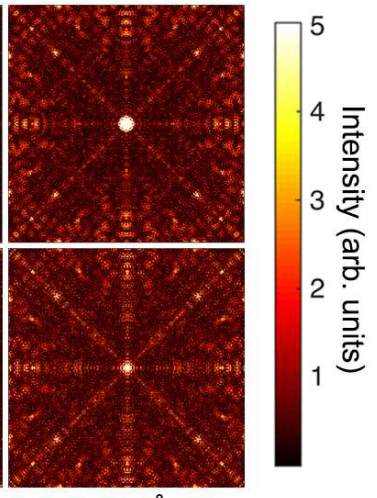

$600 \AA$

Figure 5: Distribution of the electron probe density as it channels within $\mathrm{SrTiO}_{3}$ for two convergence angles, 13.7 mrad and 19.5 mrad, with the probe positioned

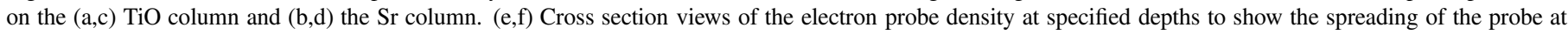
different conditions. Note: effective source size is not included in these simulations.

nificant distance away. In other words, beyond this thickness it is highly questionable as to whether the nominal gain in signal represents usefully interpretable information. Fig. 6(d) looks at the decomposition of the total $\mathrm{Sr} \mathrm{K}$-shell ionization signal with the probe placed above the $\mathrm{TiO}$ column. The TiO column is the location of the minimum in the 2D Sr-K peak EDX maps, which is sometimes regarded as the "background" or "non-columnresolved" signal in 2D STEM images [41]. However, the differences in the attribution to different columns between Figs. 6(a) and (d) suggest that the picture of column-resolved and non-column-resolved signal should not be taken too literally in quantitative work - this distinction can only be reliably established through simulation.

Figs. 6 (b), (c) and (e), (f) present an alternative way of showing how far afield some of the contributions to the total EDX signal come from for a given probe position. Two thicknesses in the range where there are significant contributions from other columns have been chosen, specifically $300 \AA$ and $600 \AA$. For both these thicknesses, the accumulated percentage contribution for both $\mathrm{Sr}-\mathrm{K}$ and $\mathrm{Ti}-\mathrm{K}$ is shown as a function of the distance away from the column on which the probe is placed. For example, Fig. 6(b) shows that, for the $19.5 \mathrm{mrad}$ probe-forming convergence semiangle, $95 \%$ of the total Sr-K signal contribution comes from columns within a fairly local- ized one unit-cell ( $3.9 \AA$ A radial distance) of the $\mathrm{Sr}$ column under the probe for a $300 \AA$ A thick sample, whereas the more delocalized three unit-cell's radial distance is needed to account for the same percentage of the total signal if the specimen is 600 $\AA$ thick. For the Sr-K off-column case, accounting for $95 \%$ of the signal involves a three unit-cell's radial distance for a 300 $\AA$ thick sample and more than four unit-cell's radial distance for a $600 \AA$ thick sample. For Ti-K, the corresponding radial distances are a little smaller, but certainly of the same order as that for Sr-K. It should be emphasised that this discussion represents a best case scenario of the probe being exactly centered on the column. Once the effective source size and integrated column signal are included, the percentage contribution of signal from more distance columns is expected to increase [32]. This is confirmed in the supplementary information, Fig. S5, for the Sr-K signal when the probe is on the Sr column.

It is interesting to note that the spatial distribution of the accumulated percentage contribution for probe-forming convergence angles in the range from 13.7 to $30 \mathrm{mrad}$ - typical of experiments on aberration-corrected instrument - are quite similar when the probe is on the atomic column of interest. Only for the very large convergence angle of $45.7 \mathrm{mrad}$ does an appreciably larger fraction of the signal come from further afield. With the probe in the signal minimum position, on the $\mathrm{TiO}$ 
(a)

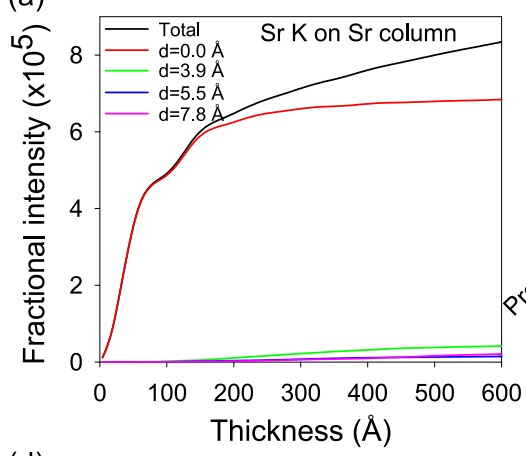

(d)

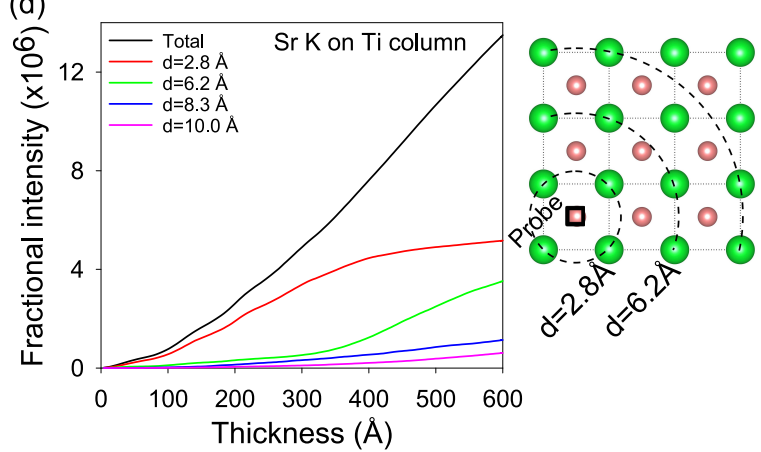

(b)

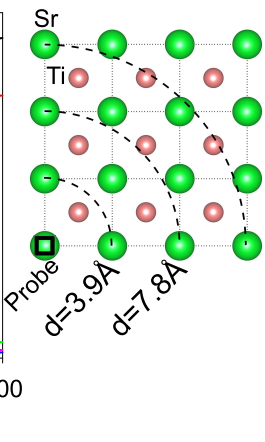

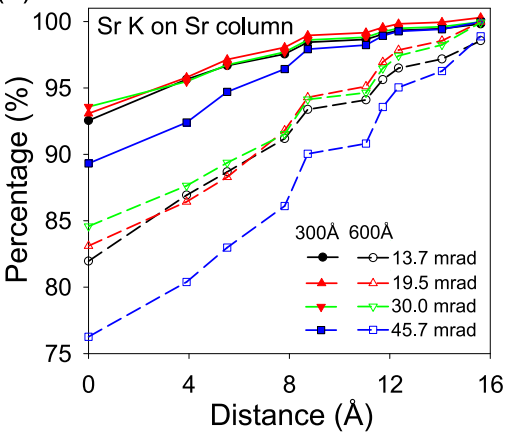

(e)

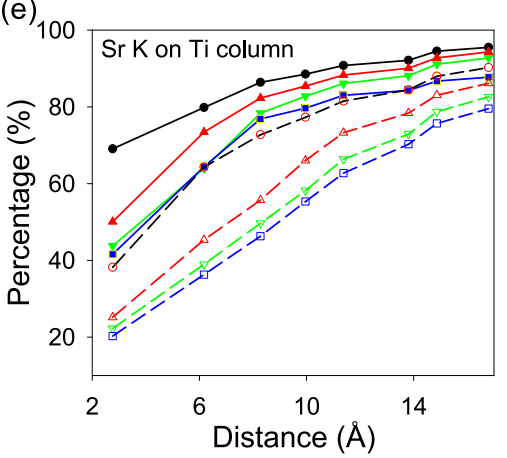

(c)

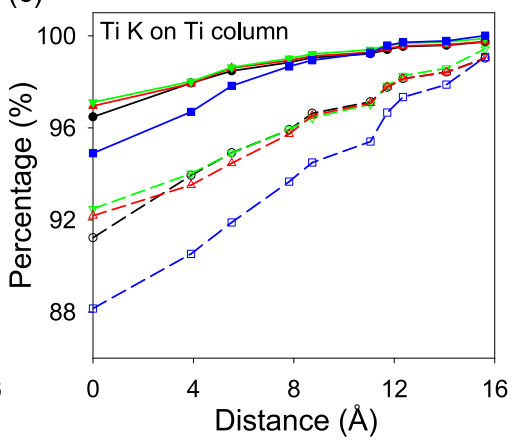

(f)

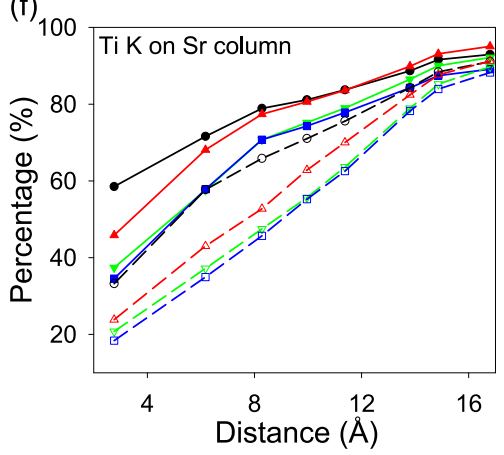

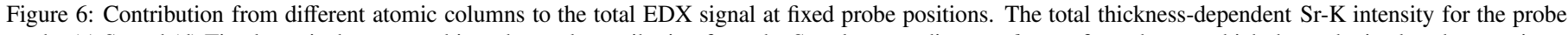
on the (a) Sr and (d) Ti column is decomposed into the total contribution from the Sr columns a distance $d$ away from that on which the probe is placed, assuming a

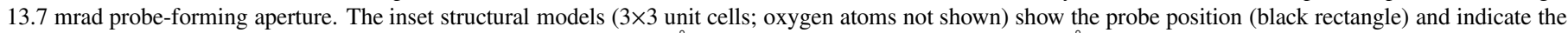
distances $d$ to nearby Sr columns. For the selected thicknesses of $300 \AA$ (solid line and filled symbols) and $600 \AA$ (dashed line and open symbols) and four different

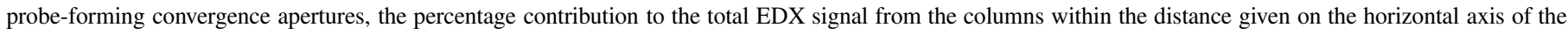
fixed probe position is shown for: (b) Sr-K signal with the probe on a Sr column; (e) Sr-K signal with the probe on a Ti column; (c) Ti-K signal for the probe on

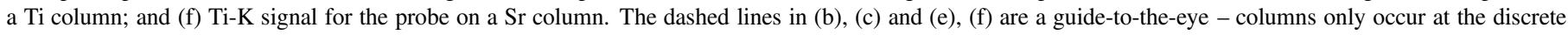
distances marked by the symbols. Note: effective source size is not included in these simulations.

column when looking at the Sr-K signal or on the Sr column when looking at the Ti-K signal, the "delocalization" of the signal, i.e. the size of the region away from the probe location in which most of the signal is generated, increases with increasing probe-forming convergence angle, qualitatively consistent with expectations for geometric probe spreading.

In summary, a relatively thin area should be chosen for quantitative EDX mapping at atomic resolution. Even then, dynamical scattering would necessitate simulations be used to reliably draw the quantitative correspondence between the number of $\mathrm{X}$ ray counts measured and the number of atoms in a column. In principle, extremely thin specimens (much less than $50 \AA$ [11]) might allow channelling to be neglected. However, in practice it would make for an extremely challenging experiment to obtain sufficient signal-to-noise in 2D maps from such samples without adverse effects from beam damage. If committing to performing simulation anyway, the interpretive complications that arise from probe spreading for larger specimen thickness could be incorporated [9] or corrected [42], assuming the structure is well known. This may be the case for perfect crystals, but it is less likely for defect structures, such as dislocation cores, interfaces and surfaces. For such cases it may be preferable to work within a domain which warrants reasonable confidence that the overwhelming majority of the recorded signal comes from the column underneath the probe.

\section{Conclusion}

In summary, generally good absolute-scale quantitative agreement between simulation and experiment in two dimensional atomic resolution EDX maps has been demonstrated. The most pronounced discrepancies, in the $\mathrm{Sr}-\mathrm{K}$ and $\mathrm{O}-\mathrm{K}$ peaks, appear to be systematic, and various technical details that could cause such discrepancies have been discussed. This analysis shows that careful characterization of the experimental set-up will be a critical factor when seeking to apply quantitative EDX analysis in practice, especially in view of the more complex detector geometry necessary to obtain sufficient X-ray counts for atomic-resolution mapping on thin samples.

For $\mathrm{SrTiO}_{3}$ and $200 \mathrm{kV}$ electrons, we suggest that samples in the thickness range 150-200 $\AA$ represent a good compromise between the competing considerations of the specimen being sufficiently thick as to provide good signal-to-noise while being sufficiently thin that column-by-column analysis is reliable. Though the notion that simple column-by-column analysis requires a thickness compromise between maximizing signal-tonoise ratio while minimizing contributions from other columns 
in general, the numerical values given here are likely specific to this material and accelerating voltage.

This work used a known structure as a test case to establish the reliability of atomic resolution 2D EDX mapping on an absolute scale. Provided that channelling effects can be either incorporated or minimized, this capability should enable measured EDX intensities to be interpreted in terms of the number of atoms present, which is especially useful for nanoscale material systems, such as nanoparticles, defects and interface structures, in which relative concentration is either not well defined or else not particularly useful. Compared with atomic counting via quantitative HAADF [43, 44], EDX mapping has several benefits. In particular, it permits unambiguous elemental analysis, even in systems with elements of similar atomic number or with more than two kinds of elements in one atomic column. The sensitivity to detect dilute dopants by EDX analysis is also potentially better if the signal-to-noise of EDX spectra can be further improved.

\section{Acknowledgements}

We acknowledge the use of facilities for sample preparation and checking in the Monash Centre for Electron Microscopy. This research was supported under the Australian Research Council's Discovery Projects funding scheme (Projects DP110102228 and DP140102538), and its DECRA funding scheme (Project DE130100739). XS, WX, JHD, and JML gratefully acknowledge the Air Force Office of Scientific Research (FA9550-14-1-0182) for support of this research and also acknowledge the Analytical Instrumentation Facility (AIF) at North Carolina State University, which is supported by the State of North Carolina and the National Science Foundation. JHD acknowledges support for this work by the National Science Foundation Graduate Research Fellowship (Grant DGE1252376).

\section{References}

\section{References}

[1] A. J. D’Alfonso, B. Freitag, D. Klenov, L. J. Allen, Atomic-resolution chemical mapping using energy-dispersive X-ray spectroscopy, Phys. Rev. B 81 (2010) 100101(R).

[2] M.-W. Chu, S. C. Liou, C.-P. Chang, S.-F. Choa, C. H. Chen, Emergent chemical mapping at atomic-column resolution by energy-dispersive $\mathrm{X}$ ray spectroscopy in an aberration-corrected electron microscope, Phys. Rev. Lett. 104 (2010) 196101

[3] M. Watanabe, M. Kanno, E. Okunishi, Atomic-resolution elemental mapping by EELS and XEDS in aberration corrected STEM, JEOL news 45 (2010) 8-15.

[4] D. O. Klenov, M. O. Zide, Structure of the InAlAs/InP interface by atomically resolved energy dispersive spectroscopy, Appl. Phys. Lett. 99 (2011) 141904.

[5] L. J. Allen, A. J. D'Alfonso, B. Freitag, D. Klenov, Chemical mapping at atomic resolution using energy-dispersive X-ray spectroscopy, MRS Bulletin 37 (2012) 47-52.

[6] P. Lu, J. Xiong, M. Van Benthem, Q. Jia, Atomic-scale chemical quantification of oxide interfaces using energy-dispersive X-ray spectroscopy, Appl. Phys. Lett. 102 (2013) 173111.

[7] J. M. LeBeau, S. D. Findlay, L. J. Allen, S. Stemmer, Quantitative atomic resolution scanning transmission electron microscopy, Phys. Rev. Lett. 100 (2008) 206101.
[8] G. Kothleitner, M. J. Neish, N. R. Lugg, S. D. Findlay, W. Grogger, F. Hofer, L. J. Allen, Quantitative elemental mapping at atomic resolution using X-ray spectroscopy, Phys. Rev. Lett. 112 (2014) 085501.

[9] B. D. Forbes, A. J. D’Alfonso, R. E. A. Williams, R. Srinivasan, H. L. Fraser, D. W. McComb, B. Freitag, D. O. Klenov, L. J. Allen, Contribution of thermally scattered electrons to atomic resolution elemental maps, Phys. Rev. B 86 (2012) 024108.

[10] J. H. Dycus, W. Xu, X. Sang, A. J. D'Alfonso, Z. Chen, M. Weyland, L. J. Allen, S. D. Findlay, J. M. LeBeau, Infuence of experimental conditions on quantitative atomic resolution energy dispersive X-ray spectroscopy, Submitted for publication.

[11] Z. Chen, A. J. D’Alfonso, M. Weyland, D. J. Taplin, S. D. Findlay, L. J. Allen, Absolute scale energy dispersive X-ray analysis in scanning transmission electron microscopy, Ultramicroscopy 157 (2015) 21-26.

[12] H. S. von Harrach, P. Dona, B. Freitag, H. Soltau, A. Niculae, M. Rohde, An integrated silicon drift detector system for FEI Schottky field emission transmission electron microscopes, Microsc. Microanal. 15 (2009) 208209.

[13] F. Yang, F. Scheltens, D. McComb, D. B. Williams, M. De Graef, Absorption corrections for a Four-Quadrant SuperX EDS detector, Microsc. Microanal. 20 (2014) 100-101.

[14] W. Z. Xu, J. H. Dycus, X. H. Sang, J. M. LeBeau, A numerical model for multiple detector energy dispersive X-ray spectroscopy in the transmission electron microscope, Ultramicroscopy 164 (2016) 51-61.

[15] S. Hillyard, R. F. Loane, J. Silcox, Annular dark-field imaging: Resolution and thickness effects, Ultramicroscopy 49 (1993) 14-25.

[16] L. J. Allen, S. D. Findlay, M. P. Oxley, C. J. Rossouw, Lattice-resolution contrast from a focused coherent electron probe. Part I, Ultramicroscopy 96 (2003) 47-63.

[17] C. Dwyer, J. Etheridge, Scattering of $\AA$-scale electron probes in silicon, Ultramicroscopy 96 (2003) 343-360.

[18] FEI company, ChemiSTEM technology: A revolution in EDX analytics (2010). URL http://www.fei.com/documents/chemistem-technology/

[19] C. M. Parish, R. M. White, J. M. LeBeau, M. K. Miller, Response of nanostructured ferritic alloys to high-dose heavy ion irradiation, J. Nucl. Mater. 445 (2014) 251-260

[20] J. M. LeBeau, S. D. Findlay, L. J. Allen, S. Stemmer, Position averaged convergent beam electron diffraction: Theory and applications, Ultramicroscopy 110 (2010) 118-125.

[21] Q. Tseng, Template matching and slice alignment - ImageJ Plugins (2011).

URL https://sites.google.com/site/qingzongtseng/ template-matching-ij-plugin

[22] T. C. Huang, Rapid and precise method for analysis of energy dispersive X-ray spectra, IBM J. Res. Develop. 23 (1979) 206-213.

[23] A. Kahoul, V. Aylikci, N. K. Aylikci, E. Cengiz, G. Apaydin, Updated database and new empirical values for K-shell fluorescence yields, Radiat. Phys. Chem. 81 (2012) 713-727.

[24] J. H. Hubbell, P. N. Trehan, N. Singh, B. Chand, D. Mehta, M. L. Garg, R. R. Garg, S. Singh, S. Puri, A review, bibliography, and tabulation of K, $\mathrm{L}$, and higher atomic shell X-ray fluorescence yields, J. Phys. Chem. Ref. Data 23 (1994) 339-364.

[25] P. Schlossmacher, D. O. Klenov, B. Freitag, S. von Harrach, A. Steinbach, Nanoscale chemical compositional analysis with an innovative S/TEMEDX system, Microscopy and Analysis 24 (2010) S5-S8.

[26] B. D. Forbes, A. V. Martin, S. D. Findlay, A. J. D'Alfonso, L. J. Allen, Quantum mechanical model for phonon excitation in electron diffraction and imaging using a Born-Oppenheimer approximation, Phys. Rev. B 82 (2010) 104103

[27] L. J. Allen, A. J. D'Alfonso, S. D. Findlay, Modelling the inelastic scattering of fast electrons, Ultramicroscopy 151 (2015) 11-22.

[28] T. J. A. Slater, A. Janssen, P. H. C. Camargo, M. G. Burke, N. J. Zaluzec, S. J. Haigh, STEM-EDX tomography of bimetallic nanoparticles: A methodological investigation, Ultramicroscopy 162 (2016) 61-73.

[29] D. O. Klenov, S. D. Findlay, L. J. Allen, S. Stemmer, Influence of orientation on the contrast of high-angle annular dark-field images of silicon, Phys. Rev. B 76 (2007) 014111

[30] C. Maunders, C. Dwyer, P. C. Tiemeijer, J. Etheridge, Practical methods for the measurement of spatial coherence - a comparative study, Ultramicroscopy 111 (2011) 1437-1446. 
[31] J. Verbeeck, A. Béché, W. Van der Broek, A holographic method to measure the source size broadening in STEM, Ultramicroscopy 120 (2012) $35-40$.

[32] D. T. Nguyen, S. D. Findlay, J. Etheridge, The spatial coherence function in scanning transmission electron microscopy and spectroscopy, Ultramicroscopy 146 (2014) 6-16.

[33] C. Dwyer, C. Maunders, C. L. Zheng, M. Weyland, P. C. Tiemeijer, J. Etheridge, Sub-0.1 nm-resolution quantitative scanning transmission electron microscopy without adjustable parameters, Appl. Phys. Lett. 100 (2013) 191915.

[34] C. J. Rossouw, C. Dwyer, H. Katz-Boon, J. Etheridge, Channelling contrast analysis of lattice images: Conditions for probe-insensitive STEM, Ultramicroscopy 136 (2014) 216-223.

[35] H. E, K. E. MacArthur, T. J. Pennycook, E. Okunishi, A. J. D’Alfonso, N. R. Lugg, L. J. Allen, P. D. Nellist, Probe integrated scattering cross sections in the analysis of atomic resolution HAADF STEM images, Ultramicroscopy 133 (2013) 109-119.

[36] J. T. Armstrong, P. R. Buseck, A general characteristic fluorescence correction for the quantitative electron microbeam analysis of thick specimens, thin films and particles, X-Ray Spectrometry 14 (1985) 172-182.

[37] G. Cliff, G. W. Lorimer, The quantitative analysis of thin specimens, J. Microscopy 103 (1975) 203-207.

[38] Y. Peng, P. D. Nellist, S. J. Pennycook, HAADF-STEM imaging with sub-angstrom probes: a full Bloch wave analysis, J. Electron Microsc. 53 (2004) 257-266.

[39] M. P. Oxley, E. C. Cosgriff, L. J. Allen, Nonlocality in imaging, Phys. Rev. Lett. 94 (2005) 203906.

[40] R. F. Egerton, Electron Energy-Loss Spectroscopy in the Electron Microscope, Third Edition, Plenum Press, New York, 2011.

[41] D. O. Klenov, S. Stemmer, Contributions to the contrast in experimental high-angle annular dark-field images, Ultramicroscopy 106 (2006) 889901.

[42] M. J. Neish, N. R. Lugg, S. D. Findlay, M. Haruta, K. Kimoto, L. J. Allen, Detecting the direction of oxygen bonding in $\mathrm{SrTiO}_{3}$, Phys. Rev. B 88 (2013) 115120.

[43] N. P. Young, Z. Y. Li, Y. Chen, S. Palomba, M. Di Vece, R. E. Palmer, Weighing supported nanoparticles: Size-selected clusters as mass standards in nanometrology, Phys. Rev. Lett. 101 (2008) 246103.

[44] J. M. LeBeau, S. D. Findlay, L. J. Allen, S. Stemmer, Standardless atom counting in scanning transmission electron microscopy, Nano Lett. 10 (2010) 4405-4408. 